\title{
Value of endoscopy with narrow-band imaging and probe- based confocal laser endomicroscopy in the diagnosis of preneoplastic lesions of gastrointestinal tract
}

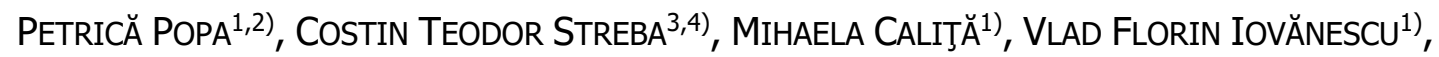



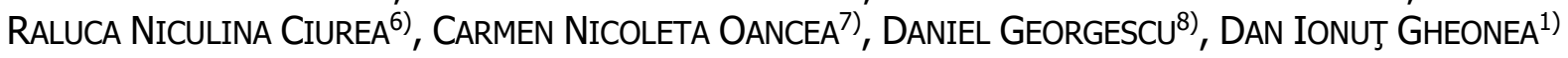 \\ 1) Department of Gastroenterology, University of Medicine and Pharmacy of Craiova, Romania \\ 2)PhD Student, Doctoral School, University of Medicine and Pharmacy of Craiova, Romania \\ 3) Department of Scientific Research Methodology, University of Medicine and Pharmacy of Craiova, \\ Romania \\ 4) Department of Pulmonology, University of Medicine and Pharmacy of Craiova, Romania \\ 5) Department of Intensive Care, University of Medicine and Pharmacy of Craiova, Romania \\ ${ }^{6}$ ) Department of Pathology, University of Medicine and Pharmacy of Craiova, Romania \\ 7) Department of Analytical Chemistry, University of Medicine and Pharmacy of Craiova, Romania \\ 8) Department of Medical Informatics and Biostatistics, University of Medicine and Pharmacy of Craiova, \\ Romania
}

\begin{abstract}
Introduction: Amongst all malignant tumors, cancers of the digestive tract rank first in terms of yearly deaths. Patients above 60 years of age are the most affected, as the diagnosis is frequently made in advanced stages of the disease when therapy is less effective. Our study aimed to evaluate the efficiency of narrow-band imaging (NBI) endoscopy and probe-based confocal laser endomicroscopy (pCLE) in the correct diagnosis of preneoplastic lesions in the upper and lower digestive tract. Patients, Materials and Methods: We included 46 patients with digestive preneoplastic lesions, who underwent either upper or lower digestive endoscopy, followed by NBI and pCLE. We recorded 5-10 frames per each lesion, from different angles and distances during white-light endoscopy and selected frames from full recordings of $\mathrm{NBI}$ and pCLE. Usual preparation was used for the endoscopic procedures; pCLE required in vivo administration of $10 \%$ Sodium Fluorescein as a contrast agent. Pathology was performed in case of solid tumors. Three medical professionals with different levels of training, blinded to the results, interpreted the data. Results: The experienced physician correlated very well the NBI findings with pathology $(0.93, p=0.05)$, while the resident physician and the experienced nurse obtain lower, albeit still statistically significant, values $(0.73$ and 0.62 , respectively). For pCLE, the experienced physician obtained near-perfect correlation with pathology (0.96), followed closely by the resident physician (0.93). The nurse obtained a modest correlation (0.42). All examiners obtained approximately equal performances in discerning between malignant and benign lesions. Conclusions: Digestive endoscopy in NBI mode proved its effectiveness. Even less experienced endoscopists can achieve good results, while an experienced nurse can positively influence the diagnosis. In the case of pCLE, when available, it can greatly reduce diagnostic times, while requiring higher expertise and specialty training.
\end{abstract}

Keywords: preneoplastic lesions, chromoendoscopy, confocal laser endomicroscopy based on mini samples.

\section{ค Introduction}

Cancers are one of the leading causes of mortality globally, regardless of the level of development of states. Of all, gastrointestinal cancers rank first in terms of the annual number of deaths [1]. The most affected patients are those in the $6^{\text {th }}$ to the $8^{\text {th }}$ decades of life [2]. Most of the times, the diagnosis is made in advanced stages of the disease when the chemotherapy is no longer effective, and the prognosis is reserved [3]. The situation is caused by both the lack of screening programs and their cost, as well as exposure to more and more toxic substances, malnutrition, lifestyle, etc. One of the main objectives in gastroenterological and oncological research is the development of new methods for the early diagnosis of premalignant and malignant lesions and their therapeutic sanctioning with much greater benefits for the patient, but also in terms of costs. Currently, there are few countries that have implemented solid screening programs to prevent digestive tract cancers. In Romania, screening is rather opportunistic and preneoplastic or malignant lesions are diagnosed following the investigation of other pathologies or syndromes. Usually, surveillance by upper digestive endoscopy (UDE) or lower digestive endoscopy (LDE) in white light is recommended depending on age, previously diagnosed lesions or diseases, heredocollateral antecedents, eating habits, etc. Conventional endoscopy cannot distinguish lesions according to histological substrate. For this reason, the histopathological (HP) examination remains the one that dictates the management of patients.

This is an open-access article distributed under the terms of a Creative Commons Attribution-NonCommercial-ShareAlike 4.0 International Public License, which permits unrestricted use, adaptation, distribution and reproduction in any medium, non-commercially, provided the new creations are licensed under identical terms as the original work and the original work is properly cited. 
The process is often difficult [4-8]. Not infrequently, the results of biopsies arrive even after four weeks of waiting, precious time especially in the management of patients with malignant lesions. Also, the biopsies taken can be inconclusive if they are not taken on purpose and many lesions can be mismanaged (unnecessary resections, unnecessary surveillance, pathology services burdened with unnecessary biopsies). An important proportion of the total number of cases of the digestive tract have as a starting point a preneoplastic lesion. Their management is often inappropriate due to the ignorance of the HP substrate of the lesions and the potential for malignancy. Thus, there was a need to develop new endoscopic techniques that would allow true "optical biopsies" by analyzing the pattern of mucosa and intra- and perilesional vessels or even more, visualization of the lesions at the cellular level. Narrow-band imaging (NBI) endoscopy and probe-based confocal laser endomicroscopy (pCLE) are both alternatives for the diagnosis of preneoplastic lesions in the upper and lower digestive tract. The availability of the two methods is totally different. If virtual chromoendoscopy is available on most new endoscopes at the push of a button and at no extra cost, endomicroscopy is still inaccessible to many centers due to the high price of equipment and consumables.

\section{Aim}

Through this study, we aimed to evaluate the effectiveness of two methods mentioned above, endoscopy in NBI mode and pCLE, both in terms of establishing the diagnosis of preneoplastic lesions and in terms of early detection of malignancies. We will also highlight the importance of experience in using each method and the learning curve to obtain optimal results. In the future, we will try to implement an instant HP diagnostic algorithm based on the computerized analysis of the obtained images.

\section{ㅁ Patients, Materials and Methods}

\section{Patients}

After obtaining clearance from the Ethics Committee of the University of Medicine and Pharmacy of Craiova, Romania, we conducted a prospective study over 24 months (January 2017-October 2019), at the Clinic of Gastroenterology and Hepatology of the University. The study included 46 known patients with preneoplastic lesions of the upper and lower digestive tract, suspected patients with typical digestive symptoms and patients diagnosed with digestive tract lesions following routine checks or prevention. The study was performed on a single group of patients with preneoplastic or malignant degenerative lesions, from the upper and lower digestive tract, who underwent endoscopy in the NBI mode and CLE. Some of the study participants had been previously diagnosed with preneoplastic lesions of the digestive tract and presented to the Clinic for endoscopic reassessment, biopsy, and the establishment of appropriate therapeutic conduct. The other patients included in the group were those presented in the Clinic of Gastroenterology with digestive symptoms, such as: dysphagia, odynophagia, heartburn, abdominal pain (localized, diffuse, intermittent, permanent), involuntary weight loss, vomiting, hemat- emesis, emission of melenic stools or hematochezia, rectorrhagia, transit disorders (constipation, diarrhea, alternating diarrhea-constipation). The exclusion criteria from the study were the following: patients who refused to sign informed consent to perform endoscopic procedures; patients allergic to the contrast substance (Fluorescein); patients who refuse sedation with Propofol or who have conditions that are incompatible with the procedure; patients undergoing cancer treatment and hemodynamic instability.

\section{Equipment and procedures}

UDE was performed in patients with symptoms typical of the upper digestive tract, after fasting for at least six hours for both fluids and solids. The explorations were performed with the GIF-H185 and GIF-HQ190 gastroscopes connected to an Olympus EVIS Exera III system. Endoscopies were performed according to the quality standards established by the American Society of Gastroenterology (ASGE). The identified lesions were examined in white light and subsequently in NBI mode. We recorded 5-10 frames per each lesion, from different angles and distances. LDE was performed in patients with symptoms typical of the lower digestive tract and in patients who presented for colorectal cancer (CRC) screening. The scans were performed with the CF-H185 colonoscope connected to an Olympus EVIS Exera III system. All colonoscopies were complete, until the visualization of the ileo-cecal valve or until the highlighting of stenoses or tumor that no longer allowed the advancement. According to $A S G E$ standards, the right colon was intubated twice, and the withdrawals lasted at least six minutes. In many cases, ENDOCUFF Olympus accessories were also used, which allowed a better visualization of the colon by exposing the mucosal folds. Colon preparation was performed the day before the colonoscopy. This involved ingesting four liters of Macrogol 4000 solution for bowel lavage. Oral feeding was also stopped from the beginning of the preparation until the colonoscopy was performed. The identified lesions were examined in white light and subsequently in NBI mode. We also took between 5-10 frames for each lesion, from different angles and distances. The endomicroscopic examination was performed in vivo and the preparation was similar to the preparation for UDE/LDE of patients (fasting for at least six hours for UDE and ingestion of osmotic solutions for intestinal lavage in case of LDE). pCLE was performed with an endomicroscopy system based on mini probes developed by Cellvizio (Mauna Kea Technologies, Paris, France) located at the Research Center for Gastroenterology and Hepatology of Craiova (Figure 1, A and B). The movements (agitated patient, vomiting reflex, peristalsis of the digestive tract, respiratory movements, and heartbeat) are the most incriminated in the formation of artifacts (Figure 2). For this reason, all patients with lesions in the upper digestive tract and a few with lesions in the lower tract who could not tolerate well the procedure were sedated with $10 \mathrm{mg} / \mathrm{mL}$ Propofol. The sedation was performed by an anesthesiologist.

We used 10\% Sodium Fluorescein as a contrast agent, in a $5 \mathrm{~mL}$ dose (one ampoule) for each patient. Examination of pCLE began approximately one minute after injection (Figure 3). 
Because the mini samples for pCLE are inserted on the working channel of the endoscope, it was done by using plastic caps (Figure 4) fixed to the distal end of the endoscope and by sucking the mucosa. Thus, the lesions of interest were more stable, and the frequency of movement artifacts was lower.
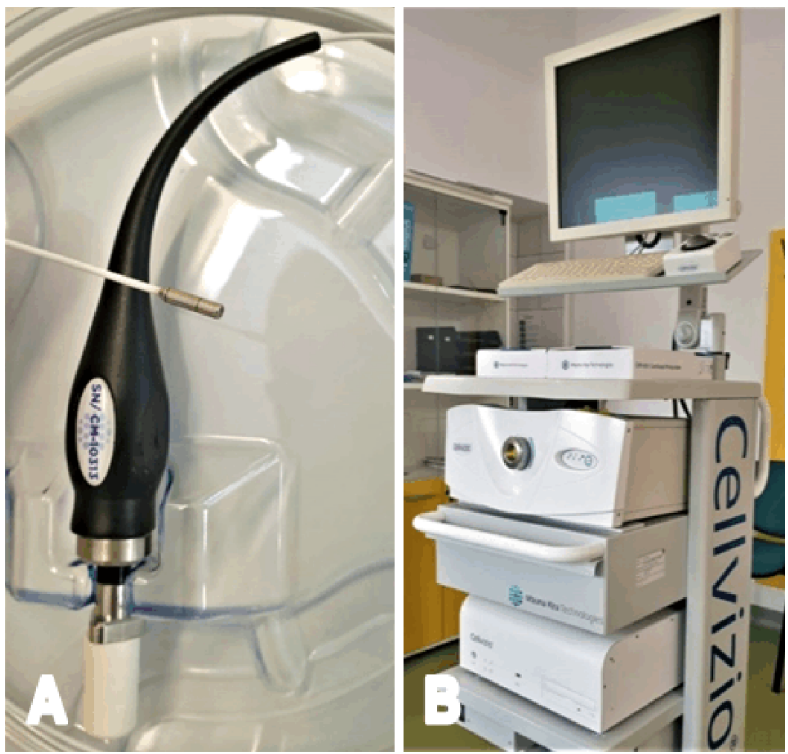

Figure 1 - (A) Mini test for the pCLE system; (B) Cellvizio pCLE system (Mauna Kea Technologies, Paris, France). pCLE: Probe-based confocal laser endomicroscopy.

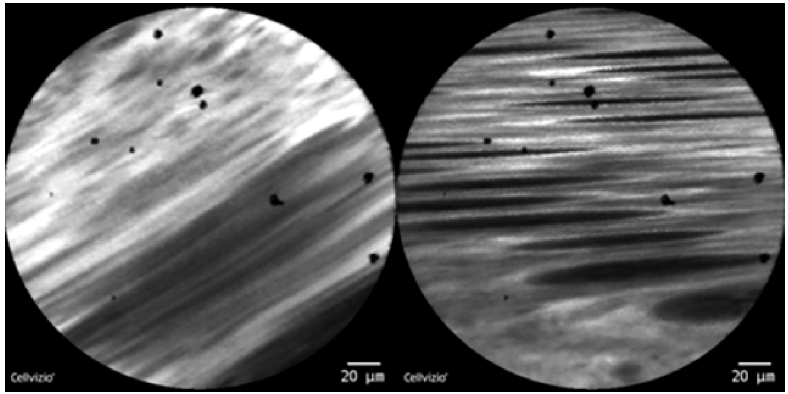

Figure 2 - Motion artifacts in pCLE assessment. pCLE: Probe-based confocal laser endomicroscopy.

In order to avoid bleeding and implicitly the artifacts, the biopsies were taken targeted after the completion of the pCLE examination. Four to eight biopsy fragments were sampled, which were fixed in formalin and embedded in paraffin. Sections were interpreted in Hematoxylin-Eosin

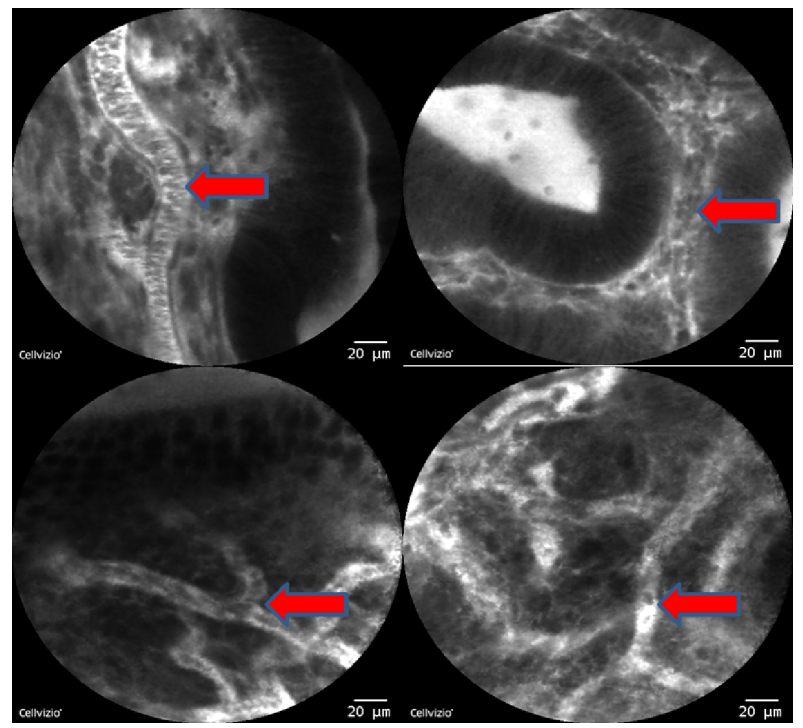

Figure 3 - Intra- and perilesional vascular structures, visualized after injection of the contrast agent. Filling gaps are blood figures that do not capture the contrast substance.

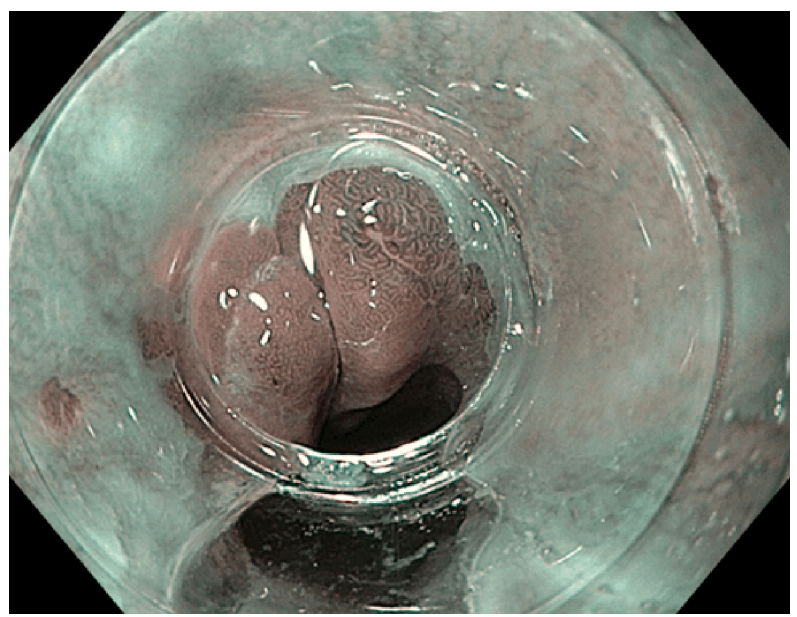

Figure 4-Plastic cap attached to the distal end of the endoscope to stabilize a short-segment EB prior to pCLE examination. EB: Endoscopic biopsy; pCLE: Probebased confocal laser endomicroscopy.

(HE) staining. For each patient, approximately 20 images were recorded, taken at 1-2 second intervals, by pressing a pedal at the examiner's foot. The images were taken both from the normal mucosa (Figure 5, A-C) and from the lesion of interest.

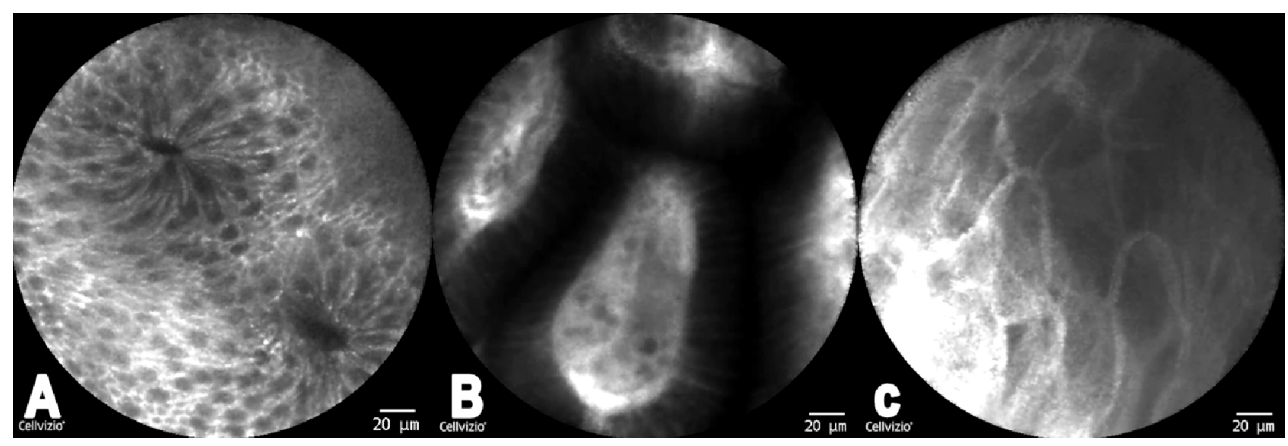

Figure 5 - pCLE examination: (A) Normal-looking colonic mucosa with round crypt architecture, regularly arranged goblet cells, and normal-looking pericytic vascular network; (B) Gastric mucosa of normal appearance, with round, regular crypts, and typical brush appearance of the cell arrangement; (C) Normal-looking squamous esophageal mucosa, flat-celled, without crypts or villi. pCLE: Probe-based confocal laser endomicroscopy. 
These were subsequently analyzed by observers with different degrees of training in Gastroenterology and in the interpretation of pCLE images: one experienced nurse, one resident physician and one primary physician. The three evaluators were not present during the real-time examination and the presumptive diagnosis for each patient was not known. Prior to the start of the study, all examiners mastered the Miami classification for pCLE, the NBI International Colorectal Endoscopic (NICE), Japan NBI Expert Team (JNET), magnifying endoscopy with NBI (ME-NBI) classifications, and the most recent work on our research was reviewed.

All biopsies or resections were analyzed by a pathologist specializing in gastrointestinal lesions.

\section{Statistical analysis}

The data were statistically processed and plotted. The Microsoft Excel package (Microsoft Corporation) with the predefined functions as well as the Data Analysis module was used. The correlation between the results of

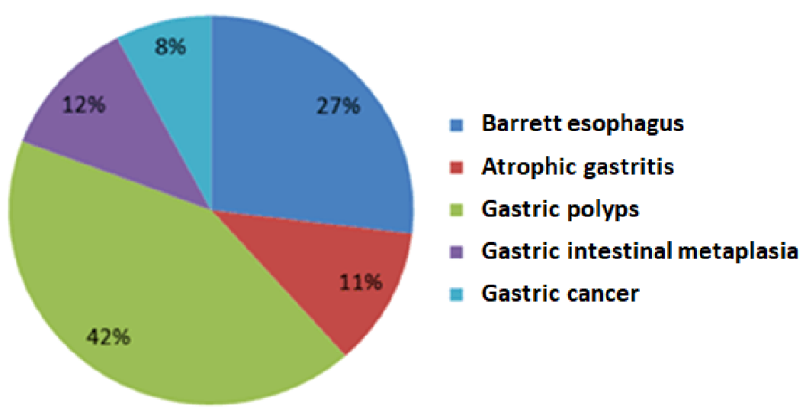

Figure 6 - Distribution of upper digestive tract lesions.

There were no allergic or adverse reactions to Fluorescein during the study.

We excluded transient yellowing of the skin and urine.

\section{NBI correlation, pCLE-HP examination for upper digestive tract lesions}

On examination of the NBI, we found that the experienced physician obtained a very close correlation with pathology $(0.93, p=0.05)$. The resident physician and the experienced nurse obtain poorer results; however, they were still within the correlation limits $(0.73$ and 0.62 , respectively). The correlation malignancy/benignity-HP examination was maximal for all examiners. Sensitivity, positive predictive value, and negative predictive value were also maximal. On pCLE examination, the experienced examiner again showed an almost maximum correlation (0.93). The resident physician achieved a performance similar to that of the NBI examination (0.71), while the experienced nurse did not fall within the correlation limits $(0.32, p>0.05)$. The experienced physician and the resident physician obtained a maximum correlation in terms of detection of malignant lesions. The experienced nurse got a correlation of only 0.45 . Sensitivity, positive predictive value, and negative predictive value were $100 \%$ for the first three. The nurse obtained $50 \%, 50 \%$ and $92 \%$, respectively (Figure 8, A-D; Figure 9, A-D). endoscopic techniques and the results of the HP examination with statistical value when $p<0.05$ was calculated. The sensitivity of NBI and pCLE for the diagnosis of each lesion was also calculated. For the detection of malignancy, the specificity, the positive predictive value, and the negative predictive value were also determined.

\section{ㅁ Results}

\section{Group characteristics}

The group of patients used for the study had an average age of 57.87 years. The minimum age was 39 and the maximum was 78 years. The distribution by gender shows a predominance of men for lower digestive tract lesions ( $75 \%$ of the total) and a slight predominance of women for upper digestive tract lesions (54\% of the total). The distribution of the studied lesions is presented in Figures 6 and 7.

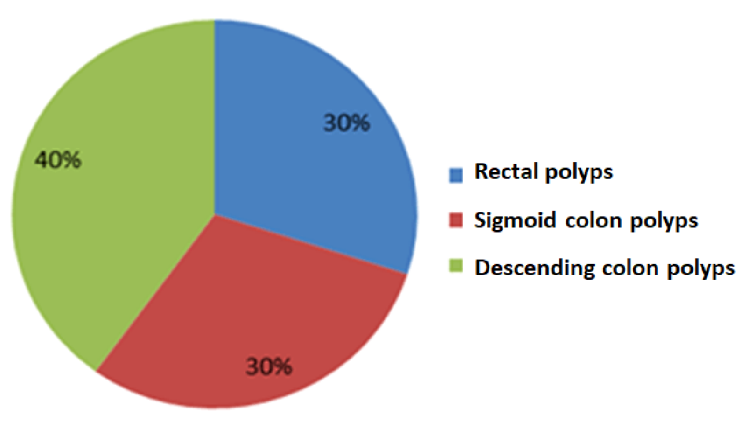

Figure 7 - Distribution of lower digestive tract lesions.

\section{NBI correlation, pCLE-HP examination for lower digestive tract lesions}

The correlation between the results of the HP exam and those of the endoscopy in NBI mode indicated a high accuracy for the primary physician $(0.96)$, as well as of the nurse (0.93). The performance of the latter emphasizes the importance of an additional experienced observer, especially when endoscopic examinations are performed by resident physicians or even young specialists. The resident physician participating in the study did not achieve similar performances, however falling within the limits of the correlation (0.56). Regarding the detection of malignant lesions, the experienced physician obtained the best correlation (0.89) and the resident physician, and the experienced nurse obtained similarly good correlations $(0.761)$. Sensitivity and positive predictive value were $100 \%$ for the experienced endoscopist and $83 \%$ for the other two evaluators. The negative predictive value was $92 \%$ for all evaluators. At the pCLE examination, the ranking of the three evaluators changed. The experienced physician obtained the best correlation (0.96), followed closely by the resident physician $(0.93)$. The nurse obtained a modest correlation (0.42). Regarding the correlation malignancy/benignity-HP examination, all examiners obtained approximately equal performances (experienced physician 0.89, experienced nurse 0.89, and the resident physician 0.80 ). Sensitivity and positive 
predictive values were $100 \%$ for all examiners. The negative predictive value was $92 \%$ for the experienced

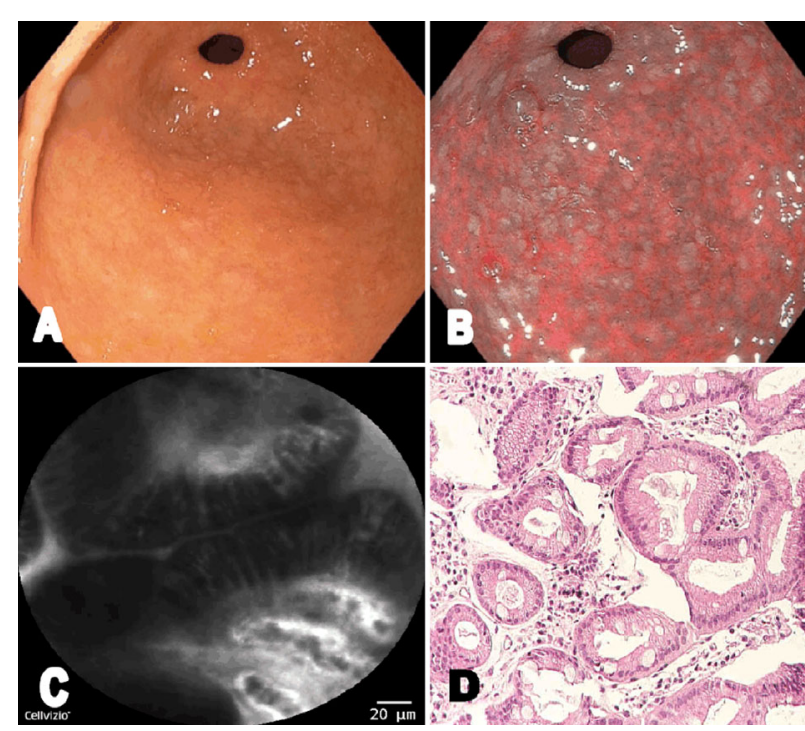

Figure 8 - Gastric intestinal metaplasia: $(A)$ White-light examination; (B) NBI mode examination; (C) pCLE examination; (D) HP examination (HE staining, $\times 100)$. HE: Hematoxylin-Eosin; HP: Histopathological; NBI: Narrow-band imaging; $p C L E$ : Probe-based confocal laser endomicroscopy.

\section{ㅁ Discussions}

Upper and lower digestive tract cancers are ideal candidates for inclusion in screening programs especially due to the existence of endoscopic diagnostic and treatment techniques that are constantly evolving. Endoscopy plays a crucial role in diagnosing preneoplastic lesions of the digestive tract and more importantly, offers the chance of detecting cancer in curative stages. The risks associated with diagnostic endoscopy are minimal and the population's access to procedures is increasing. For comparison, Japan has about 30000 endoscopists serving 127 million citizens and the United Kingdom, with perhaps the best medical system in Europe, has only 3500 endoscopists for a population of about 55 million. In Europe, there are currently no clear examination protocols for the detection of preneoplastic lesions of the upper digestive tract or quality standards for UDE, such as those for colonoscopy. Endoscopists are trained more for the detection of major and common pathologies, such as esophagitis, peptic ulcer or advanced cancers, and not for the detection of minimal changes in the digestive mucosa. In this sense, a protocol for endoscopic analysis of the stomach was implemented, which provides for the taking of eight photographs during the procedure for a new post-procedural analysis. In contrast, the Japanese protocol considers 20 captures are necessary to analyze the entire gastric mucosa, 3-4 for each area of interest. It is necessary to pay extra attention to the visualization of areas predisposed to the development of neoplasms or difficult to examine, such as the upper esophagus, right hemisphere of the lower esophagus, gastroesophageal junction or atrophic gastric mucosa. The sedation of patients is important because low tolerance for endoscopic procedures can negatively influence the outcome of the endoscopist and the nurse, respectively, and $85 \%$ for the resident physician.

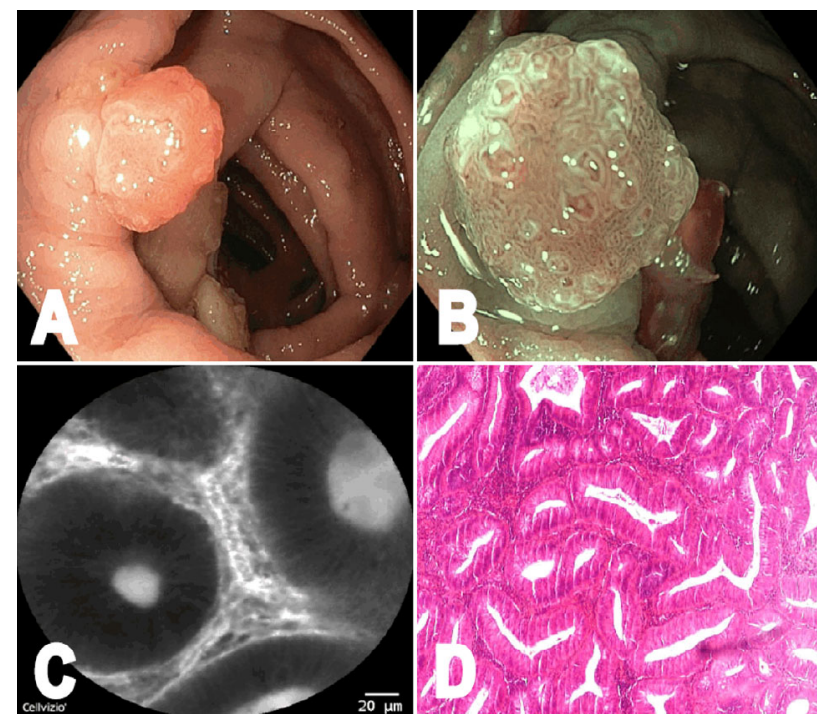

Figure 9 - Tubulo-villous colonic polyp: (A) White-light examination; (B) NBI mode examination; (C) pCLE examination; (D) HP examination (HE staining, $\times 100)$. HE: Hematoxylin-Eosin; HP: Histopathological; NBI: Narrow-band imaging; pCLE: Probe-based confocal laser endomicroscopy.

scan. Any endoscopy performed for diagnostic purposes must last a minimum of eight minutes: two minutes until advancement into the duodenum II, four minutes for examination of the gastric mucosa and two minutes for examination of the esophagus [9]. Regarding the examination of the lower digestive tract, the protocols are much clearer and mastered by many more gastroenterologists. Although the learning curve of colonoscopy is incomparably greater compared to UDE, the recognition of lesions at risk and their management seems to be assimilated more quickly by clinicians. This fact is proved by our research, where a resident physician and a nurse, additional observer to many procedures, had similar results with the experienced physician. Whether we are talking about the examination of the upper or lower digestive tract, the first step in detecting preneoplastic lesions is to visualize them in white light: discolored areas of mucosa, excavated or elevated areas of mucosa. New endoscopy systems that provide a high-definition image make this much easier. For the second time that requires the characterization of the lesions and implicitly the establishment of the diagnosis and prognosis, the virtual chromoendoscopy techniques were developed. In the upper digestive tract, in terms of detection of preneoplastic lesions, the results are impossible to ignore. In order to highlight high-grade dysplasia in patients with Barrett's esophagus, the use of chromoendoscopic techniques and targeted biopsy collection proved superior to the Seattle protocol, both in terms of HP results and in terms of overloading pathological anatomy services [10]. In the case of gastric mucosa examination, classical white-light endoscopy does not appear to be sufficient to characterize subtle mucosal changes and the use of the NBI mode may be helpful [11]. It should be noted that the normal gastric mucosa has different aspects when examining the NBI, depending 
on the area examined. The mucosa of the gastric body is characterized by small round areas surrounded by a capillary network with the appearance of honey figures. The mucosa of the antrum has regular, oval glands with central vascularization [12]. Endoscopy in NBI mode has also been shown to be effective in the diagnosis of gastric intestinal metaplasia [13]. In a multicenter study of approximately 580 patients, intestinal metaplasia was described in $7 \%$ of patients when endoscopic examination classic, in white light, respectively $17 \%$ when examined in NBI mode [14]. Areas of white-opaque mucosa on examination in NBI mode are suspicious for neoplasia and require attention [15]. In the colon, in NBI mode, the normal colonic mucosa is characterized by a circular and regular pattern of the glands and vessels. The inflamed mucosa has the same characteristics but with thicker and denser vessels. Studies have shown that NBI colonoscopic examination significantly improves the detection rate of adenomas compared to evaluation in white or highdefinition (HD) light [16-21]. The efficiency is comparable to endoscopy with classical chromoendoscopy, in the detection of adenomas and the identification of other lesions [22]. Total NBI colonoscopy is especially recommended in the examination of patients at high risk of developing CRC, Lynch syndrome and polyposis syndromes [23-25].

The results in differentiating neoplastic and nonneoplastic colonic polyps were obtained by describing the vascular pattern. It is predictive of the HP stage and even of the level of invasion of cancers in the submucosa [26-30]. It showed a sensitivity in differentiating malignant lesions from benign ones of $97.5 \%$, equal to that of ME-NBI, which associates NBI examination with magnification. However, current classifications do not include serrated polyps which are often difficult to differentiate from hyperplastic or adenomatous ones. Consequently, the Workgroup serrAted polypS and Polyposis (WASP) classification was developed that improved the endoscopic diagnosis of serous polyps, but more studies are still needed to prove its effectiveness [31-33]. The use of standardized scales improves diagnostic accuracy and significantly reduces interobserver variability.

Virtual chromoendoscopy is much easier to use compared to conventional dye-based chromoendoscopy but is not used by all clinicians who have it. The situation is caused in most cases by their perception of the training required to use the technique and the additional time required for the examination. We are currently trying to implement a new approach in the management of polyps $<5 \mathrm{~mm}$ based on the so-called optical biopsies obtained by using high-performance endoscopic techniques, without causing the multiplication of interval cancers. Studies have shown that endoscopy in NBI mode can support the strategies of "diagnose and leave" and "resect and discard" but only in the hands of an experienced endoscopist, as evidenced by the present study [34-36].

For our study, we used endoscopy in NBI mode available on the new Olympus endoscopy systems. The results obtained for the evaluation of the upper digestive tract complied with the level of training of the evaluators. The correlation of the NBI analysis with the HP examination had values increasing from 0.62 for the experienced nurse to 0.73 for the resident physician and 0.93 for the experienced gastroenterologist. Probably the most important aspect is the fact that no evaluator, regardless of the degree of training, did not underdiagnose a malignant lesion and did not negatively influence the subsequent management. Although the correlation with HP examination, sensitivity, positive predictive value, and negative predictive value had maximum values, it is necessary to extend the study to validate the results as the number of patients with malignant degenerative lesions was very small. The appearance is also valid for the sensitivity of NBI endoscopy in the diagnosis of lesions with high degree dysplasia, intestinal metaplasia, and gastric mucosal atrophy. Exceptions are hyperplastic lesions and those with low-grade dysplasia, where the values obtained may already have statistical significance. Regarding the lower digestive tract, the correlation of the HP examination with the NBI analysis did not respect the level of training of the evaluators. Consequently, the nurse obtained a correlation almost identical to that of the experienced physician (0.93/0.96) and the resident physician was within the limits of the correlation, but with a value at its lower limit. The explanation may be the nurse's experience and last but not least the lesser variability of the lesions commonly encountered in daily practice at colonoscopic evaluations. From the point of view of the detection of malignant lesions, the performances were not similar to the analysis of upper digestive tract lesions. The difference was made by a single patient with a benign lesion that was classified as malignant by the experienced physician. Again, we emphasize that no lesions analyzed by NBI were underdiagnosed, the correlation being very high anyway.

To differentiate the types of polyps, the experienced physician obtained a sensitivity of $100 \%$, but, as in the case of lesions of the upper digestive tract, additional samples are needed. The present study will be extended to a larger number of patients and the efficiency of the method, interobserver variability and the role of an additional evaluator will be much more strongly supported.

Unlike the NBI technique, available at the touch of a button, the other method of endoscopic analysis, pCLE, requires complex imaging equipment, image processing and still very expensive consumables to be used in daily medical practice. It is an important leap in the development of endoscopy, and the technique of live biopsies has so far proved its effectiveness in several studies, but the success of the examination is not always guaranteed. Experience in the use of equipment and in the interpretation of images proves to be important especially at the first examinations.

In terms of the learning curve, a 2011 study found that new users quickly gain the ability to interpret images obtained with similar efficiency to experienced users [37]. The hypothesis is also supported by the results of our research, where the resident physician, without experience in using the technique and interpreting the images obtained results almost similar to the experienced physician.

The first study that compared the results obtained at the HP examination with the results of the examination with the endomicroscope, was performed by Kiesslich et al., when the first classification of the observed changes was established. The study analyzed 13020 images from 
the examination of 390 lesions. Compared to the classical HP examination, an accuracy of $99.2 \%$ was obtained, with a sensitivity and specificity of $97.4 \%$ and $99.4 \%$, respectively [38].

Interpretation of images resulting from endomicroscopic analysis is an impediment to the use of this technique due to the limited knowledge of clinicians in the interpretation of HP images [39]. The study aimed in addition to evaluating the effectiveness in the correct diagnosis of preneoplastic lesions and highlighting the importance of clinical experience. Although the number of patients included in the study is not very high, as in the case of endoscopy in NBI mode, we aim to continue the study on a larger group to validate the results obtained. In addition, we want the images obtained to be analyzed with a computer analysis software, with the storage of parameters that provide an automatic diagnosis in the future. This eliminates the subjective factor and interobserver variability. For upper gastrointestinal lesions, endomicroscopy has so far shown, according to several studies, a specificity and sensitivity of $56 \%$ and $100 \%$ in the diagnosis of Barrett's esophagus, $88 \%$ and $90.9 \%$ in the case of gastric intestinal metaplasia and an accuracy of $97 \%$ in diagnosis of gastric adenocarcinomas in early forms.

The results of our study differ significantly depending on the location of the lesions.

For the upper digestive tract, the correlation of the HP examination result with the result of the pCLE examination is almost maximum (0.925) for the experienced gastroenterologist, with training in endomicroscopy. Also, the resident physician obtained a good correlation (0.717) if we refer to the minimum experience in endomicroscopy. The nurse's results do not fall within the correlation limits (0.321). Minimum knowledge of histology and high variability of the examined lesions are probably the main causes that can be incriminated. The trend is also observed in terms of the detection rate of malignant degenerative lesions. The correlation was in this case maximum for both the experienced physician and the resident physician. The nurse does not fall within the limit of high correlation (0.45). The results obtained after the examination of the lower digestive tract showed the same significant differences compared to the level of training of the examiners. The resident physician and the experienced examiner approach the maximum correlation, while the nurse does not fall within the limits of high correlation. For the diagnosis of malignant lesions, all examiners obtained satisfactory results, close to the maximum correlation. It should be noted that in this case the nurse obtained a higher correlation value compared to the resident physician and equal to the experienced physician.

Endomicroscopy obtained better results in the characterization of colonic polyps and in the differentiation of the neoplastic ones from the non-neoplastic ones compared to the virtual chromoendoscopy ( $91 \%$ and $77 \%$, respectively). In our research, the results are comparable in terms of sensitivity in detecting the degree of dysplasia, except for high-grade dysplasia, where pCLE proved superior: NBI (100\% for malignancy, $66 \%$ for high-grade dysplasia, $100 \%$ for low-grade dysplasia, $100 \%$ for hyperplasia),
pCLE (100\% for malignancy, $83 \%$ for high-grade dysplasia, $100 \%$ for low-grade dysplasia, $100 \%$ for hyperplasia). Due to these performances, pCLE can be a real help in implementing the "view and leave" or "resect and throw" strategy.

\section{ㅁ Conclusions}

Digestive endoscopy in NBI mode available on stateof-the-art endoscopy systems proved its effectiveness in our study and promises good results even in the hands of a less experienced endoscopist. The additional observer, in this case an experienced nurse, can positively influence the diagnosis in the case of digestive endoscopy in NBI mode, if the main examiner is less experienced. On the other hand, pCLE is a revolutionary method of diagnosing preneoplastic and neoplastic lesions of the digestive tract, with results almost similar to the HP exam, when used by a gastroenterologist trained in the use of the technique. The learning curve of pCLE is not very large and the results of an uninitiated user are close to those of an experienced physician. However, the results of pCLE still have an important variability depending on the experience of the examining physician. It is necessary to implement an image analysis algorithm to reduce interobserver variability.

\section{Conflict of interests}

The authors declare that they have no conflict of interests.

\section{Authors' contribution}

Petrică Popa and Carmen Nicoleta Oancea contributed equally to this article and share main authorship.

\section{References}

[1] Najafi F, Mozaffari HR, Karami M, Izadi B, Tavvafzadeh R, Pasdar $Y$. Trends in incidence of gastrointestinal tract cancers in Western Iran, 1993-2007. Iran Red Crescent Med J, 2011, 13(11):805-810. PMID: 22737419 PMCID: PMC3371889

[2] Enzinger PC, Mayer RJ. Gastrointestinal cancer in older patients. Semin Oncol, 2004, 31(2):206-219. https://doi.org/10.1053/ j.seminoncol.2003.12.031 PMID: 15112151

[3] Gheonea DI, Cârţână T, Ciurea T, Popescu C, Bădărău A, Săftoiu A. Confocal laser endomicroscopy and immunoendoscopy for real-time assessment of vascularization in gastrointestinal malignancies. World J Gastroenterol, 2011, 17(1):21-27. https://doi.org/10.3748/wjg.v17.i1.21 PMID: 21218080 PMCID: PMC3016676

[4] Nakai Y, Isayama H, Shinoura S, Iwashita T, Samarasena JB, Chang KJ, Koike K. Confocal laser endomicroscopy in gastrointestinal and pancreatobiliary diseases. Dig Endosc, 2014, 26(Suppl 1):86-94. https://doi.org/10.1111/den.12152 PMID: 24033351

[5] André B, Vercauteren T, Buchner AM, Krishna M, Ayache N, Wallace MB. Software for automated classification of probebased confocal laser endomicroscopy videos of colorectal polyps. World J Gastroenterol, 2012, 18(39):5560-5569. https:// doi.org/10.3748/wjg.v18.i39.5560 PMID: 23112548 PMCID: PMC3482642

[6] Zauber AG, Winawer SJ, O'Brien MJ, Lansdorp-Vogelaar I, van Ballegooijen M, Hankey BF, Shi W, Bond JH, Schapiro M, Panish JF, Stewart ET, Waye JD. Colonoscopic polypectomy and long-term prevention of colorectal-cancer deaths. N Engl J Med, 2012, 366(8):687-696. https://doi.org/10.1056/NEJM oa1100370 PMID: 22356322 PMCID: PMC3322371

[7] Winawer SJ, Zauber AG, Fletcher RH, Stillman JS, O'Brien MJ, Levin B, Smith RA, Lieberman DA, Burt RW, Levin TR, Bond JH, Brooks D, Byers T, Hyman N, Kirk L, Thorson A, Simmang C, Johnson D, Rex DK. Guidelines for colonoscopy surveillance 
after polypectomy: a consensus update by the US MultiSociety Task Force on Colorectal Cancer and the American Cancer Society. CA Cancer J Clin, 2006, 56(3):143-159; quiz 184-185. https://doi.org/10.3322/canjclin.56.3.143 PMID: 16737947

[8] Seref Köksal A, Yıldız H, Taşkıran I, Turhan N, Oztaş E, Torun S, Kayaçetin E. Low magnification narrow band imaging by inexperienced endoscopists has a high accuracy in differentiation of colon polyp histology. Clin Res Hepatol Gastroenterol, 2014, 38(6):763-769. https://doi.org/10.1016/ j.clinre.2014.05.010 PMID: 25017818

[9] Ballester V, Cruz-Correa M. Endoscopic surveillance of gastrointestinal premalignant lesions: current knowledge and future directions. Curr Opin Gastroenterol, 2014, 30(5):477483. https://doi.org/10.1097/MOG.0000000000000090 PMID: 25003602 PMCID: PMC4329641

[10] Sharma P, Hawes RH, Bansal A, Gupta N, Curvers W, Rastogi A, Singh M, Hall M, Mathur SC, Wani SB, Hoffman B, Gaddam S, Fockens P, Bergman JJ. Standard endoscopy with random biopsies versus narrow band imaging targeted biopsies in Barrett's oesophagus: a prospective, international randomised controlled trial. Gut, 2013, 62(1):15-21. https:// doi.org/10.1136/gutinl-2011-300962 PMID: 22315471

[11] Cassier PA, Ducimetière $F$, Lurkin $A$, Ranchère-Vince $D$, Scoazec JY, Bringuier PP, Decouvelaere AV, Méeus P, Cellier D, Blay JY, Ray-Coquard I. A prospective epidemiological study of new incident GISTs during two consecutive years in Rhône Alpes region: incidence and molecular distribution of GIST in a European region. Br J Cancer, 2010 103(2):165-170. https://doi.org/10.1038/sj.bjc.6605743 PMID: 20588273 PMCID: PMC2906738

[12] Muto M, Yao K, Kaise M, Kato M, Uedo N, Yagi K, Tajiri H. Magnifying endoscopy simple diagnostic algorithm for early gastric cancer (MESDA-G). Dig Endosc, 2016, 28(4):379393. https://doi.org/10.1111/den.12638 PMID: 26896760

[13] Ang TL, Pittayanon R, Lau JY, Rerknimitr R, Ho SH, Singh R, Kwek AB, Ang DS, Chiu PW, Luk S, Goh KL, Ong JP, Tan JY, Teo EK, Fock KM. A multicenter randomized comparison between high-definition white light endoscopy and narrow band imaging for detection of gastric lesions. Eur J Gastroenterol Hepatol, 2015, 27(12):1473-1478. https://doi.org/10.1097/ MEG.0000000000000478 PMID: 26426836

[14] Li H, Hou X, Lin R, Fan M, Pang S, Jiang L, Liu Q, Fu L. Advanced endoscopic methods in gastrointestinal diseases: a systematic review. Quant Imaging Med Surg, 2019, 9(5): 905-920. https://doi.org/10.21037/qims.2019.05.16 PMID: 31281783 PMCID: PMC6571190

[15] Boeriu A, Boeriu C, Drasovean S, Pascarenco O, Mocan S, Stoian M, Dobru D. Narrow-band imaging with magnifying endoscopy for the evaluation of gastrointestinal lesions. World J Gastrointest Endosc, 2015, 7(2):110-120. https:// doi.org/10.4253/wjge.v7.i2.110 PMID: 25685267 PMCID: PMC4325307

[16] Saito K, Arai K, Mori M, Kobayashi R, Ohki I. Effect of Helicobacter pylori eradication on malignant transformation of gastric adenoma. Gastrointest Endosc, 2000, 52(1):27-32. https://doi.org/10.1067/mge.2000.106112 PMID: 10882958

[17] Tomasulo J. Gastric polyps. Histologic types and their relationship to gastric carcinoma. Cancer, 1971, 27(6):13461355. https://doi.org/10.1002/1097-0142(197106)27:6<1346 :aid-cncr2820270612>3.0.co;2-v PMID: 5088211

[18] Paski SC, Semrad CE. Small bowel tumors. Gastrointest Endosc Clin N Am, 2009, 19(3):461-479. https://doi.org/ 10.1016/j.giec.2009.04.012 PMID: 19647652

[19] Borch K, Ahrén B, Ahlman H, Falkmer S, Granérus G, Grimelius L. Gastric carcinoids: biologic behavior and prognosis after differentiated treatment in relation to type. Ann Surg, 2005, 242(1):64-73. https://doi.org/10.1097/01.sla.000 0167862.52309.7d PMID: 15973103 PMCID: PMC1357706

[20] Burkitt MD, Pritchard DM. Review article: Pathogenesis and management of gastric carcinoid tumours. Aliment Pharmaco Ther, 2006, 24(9):1305-1320. https://doi.org/10.1111/j.1365 -2036.2006.03130.x PMID: 17059512

[21] Bordi C, Falchetti A, Azzoni C, D'Adda T, Canavese G, Guariglia A, Santini D, Tomassetti P, Brandi ML. Aggressive forms of gastric neuroendocrine tumors in multiple endocrine neoplasia type I. Am J Surg Pathol, 1997, 21(9):1075-1082. https://doi.org/10.1097/00000478-199709000-00012 PMID: 9298884
[22] Yeung TM, Mortensen NJ. Advances in endoscopic visualization of colorectal polyps. Colorectal Dis, 2011, 13(4):352359. https://doi.org/10.1111/j.1463-1318.2009.02142.x PMID: 19930149

[23] Rex DK, Helbig CC. High yields of small and flat adenomas with high-definition colonoscopes using either white light or narrow band imaging. Gastroenterology, 2007, 133(1):42-47. https://doi.org/10.1053/j.gastro.2007.04.029 PMID: 17631129

[24] Adler A, Aschenbeck J, Yenerim T, Mayr M, Aminalai A, Drossel R, Schröder A, Scheel M, Wiedenmann B, Rösch T. Narrow-band versus white-light high definition television endoscopic imaging for screening colonoscopy: a prospective randomized trial. Gastroenterology, 2009, 136(2):410-416.e1; quiz 715. https://doi.org/10.1053/j.gastro.2008.10.022 PMID: 19014944

[25] Adler A, Pohl H, Papanikolaou IS, Abou-Rebyeh H, Schachschal G, Veltzke-Schlieker W, Khalifa AC, Setka E, Koch M, Wiedenmann B, Rösch T. A prospective randomised study on narrow-band imaging versus conventional colonoscopy for adenoma detection: does narrow-band imaging induce a learning effect? Gut, 2008, 57(1):59-64. https://doi.org/10. 1136/gut.2007.123539 PMID: 17681999

[26] Ciurea RN, Rogoveanu I, Pirici D, Târtea GC, Streba CT, Florescu C, Cătălin B, Puiu I, Târtea EA, Vere CC. B2 adrenergic receptors and morphological changes of the enteric nervous system in colorectal adenocarcinoma. World $\mathrm{J}$ Gastroenterol, 2017, 23(7):1250-1261. https://doi.org/10.3748/ wjg.v23.i7.1250 PMID: 28275305 PMCID: PMC5323450

[27] Streba L, Forţofoiu MC, Popa C, Ciobanu D, Gruia CL, Mogoantă SŞ, Streba CT. A pilot study on the role of fractal analysis in the microscopic evaluation of colorectal cancers. Rom J Morphol Embryol, 2015, 56(1):191-196. PMID: 25826505

[28] Ciocalteu A, Gheonea DI, Saftoiu A, Streba L, Dragoescu NA, Tenea-Cojan TS. Current strategies for malignant pedunculated colorectal polyps. World J Gastrointest Oncol, 2018, 10(12): 465-475. https://doi.org/10.4251/wjgo.v10.i12.465 PMID: 30595800 PMCID: PMC6304302

[29] Becker V, von Delius S, Bajbouj M, Karagianni A, Schmid RM, Meining A. Intravenous application of fluorescein for confocal laser scanning microscopy: evaluation of contrast dynamics and image quality with increasing injection-to-imaging time. Gastrointest Endosc, 2008, 68(2):319-323. https://doi.org/ 10.1016/j.gie.2008.01.033 PMID: 18436217

[30] Meining A, Frimberger E, Becker V, Von Delius S, Von Weyhern $\mathrm{CH}$, Schmid RM, Prinz C. Detection of cholangiocarcinoma in vivo using miniprobe-based confocal fluorescence microscopy. Clin Gastroenterol Hepatol, 2008, 6(9):1057-1060. https://doi.org/10.1016/j.cgh.2008.04.014 PMID: 18639496

[31] East JE, Vleugels JL, Roelandt $P$, Bhandari $P$, Bisschops R, Dekker E, Hassan C, Horgan G, Kiesslich R, LongcroftWheaton G, Wilson A, Dumonceau JM. Advanced endoscopic imaging: European Society of Gastrointestinal Endoscopy (ESGE) Technology Review. Endoscopy, 2016, 48(11):10291045. https://doi.org/10.1055/s-0042-118087 PMID: 27711949

[32] Kamiński MF, Hassan C, Bisschops R, Pohl J, Pellisé M, Dekker E, Ignjatovic-Wilson A, Hoffman A, Longcroft-Wheaton G, Heresbach D, Dumonceau JM, East JE. Advanced imaging for detection and differentiation of colorectal neoplasia: European Society of Gastrointestinal Endoscopy (ESGE) Guideline. Endoscopy, 2014, 46(5):435-449. https://doi.org/10.1055/s0034-1365348 PMID: 24639382

[33] IJspeert JEG, Bastiaansen BAJ, van Leerdam ME, Meijer GA, van Eeden S, Sanduleanu S, Schoon EJ, Bisseling TM, Spaander MC, van Lelyveld N, Bargeman M, Wang J, Dekker E; Dutch Workgroup serrAted polypS \& Polyposis (WASP). Development and validation of the WASP classification system for optical diagnosis of adenomas, hyperplastic polyps and sessile serrated adenomas/polyps. Gut 2016, 65(6):963-970. https://doi.org/10.1136/gutjnl-2014-308411 PMID: 25753029

[34] ASGE Technology Committee; Abu Dayyeh BK, Thosani N, Konda V, Wallace MB, Rex DK, Chauhan SS, Hwang JH, Komanduri S, Manfredi M, Maple JT, Murad FM, Siddiqui UD, Banerjee S. ASGE Technology Committee systematic review and meta-analysis assessing the ASGE PIVI thresholds for adopting real-time endoscopic assessment of the histology of diminutive colorectal polyps. Gastrointest Endosc, 2015, 81(3):502.e1-502.e16. https://doi.org/10.1016/j.gie.2014.12. 022 PMID: 25597420 
[35] McGill SK, Evangelou E, loannidis JPA, Soetikno RM, Kaltenbach T. Narrow band imaging to differentiate neoplastic and non-neoplastic colorectal polyps in real time: a metaanalysis of diagnostic operating characteristics. Gut, 2013 , 62(12):1704-1713. https://doi.org/10.1136/gutjnl-2012-303965 PMID: 23300139 PMCID: PMC3841766

[36] Solon C, Klausnitzer R, Blissett D, Ihara Z. Economic value of narrow band imaging versus white light endoscopy for the characterization of diminutive polyps in the colon: systematic literature review and cost-consequence model. J Med Econ, 2016, 19(11):1040-1048. https://doi.org/10.1080/13696998. 2016.1192550 PMID: 27207009

[37] Buchner AM, Gomez V, Heckman MG, Shahid MW, Achem S, Gill KR, Jamil LH, Kahaleh M, Lo SK, Picco M, RiegertJohnson D, Raimondo M, Sciemeca D, Wolfsen H, Woodward T,
Wallace MB. The learning curve of in vivo probe-based confocal laser endomicroscopy for prediction of colorectal neoplasia. Gastrointest Endosc, 2011, 73(3):556-560. https://doi.org/10. 1016/j.gie.2011.01.002 PMID: 21353852

[38] Kiesslich R, Burg J, Vieth M, Gnaendiger J, Enders M, Delaney P, Polglase A, McLaren W, Janell D, Thomas S, Nafe B, Galle PR, Neurath MF. Confocal laser endoscopy for diagnosing intraepithelial neoplasias and colorectal cancer in vivo. Gastroenterology, 2004, 127(3):706-713. https://doi. org/10.1053/j.gastro.2004.06.050 PMID: 15362025

[39] Streba CT, Gîltan AM, Gheonea IA, Demetrian A, Şoimu AV, Săftoiu A, Gruionu G, Gruionu LG. Utility of confocal laser endomicroscopy in pulmonology and lung cancer. Rom $\mathrm{J}$ Morphol Embryol, 2016, 57(4):1221-1227. PMID: 28174787

\section{Corresponding author}

Costin Teodor Streba, Associate Professor, MD, PhD, Department of Scientific Research Methodology and Department of Pulmonology, University of Medicine and Pharmacy of Craiova, 2 Petru Rareş Street, 200349 Craiova, Romania; Phone +40722-389 906, e-mail: costin.streba@umfcv.ro

Received: June 30, 2020

Accepted: February 22, 2021 